\title{
Multi-Frequency HF Observations of Farley-Buneman Phase Velocities
}

\author{
D.R. Moorcroft, L.M. Kagan* and R.S. Kissack
} Department of Physics and Astronomy, University of Western Ontario, 1151 Richmond St., London ON, N6A 3K7,
Canada

\begin{abstract}
With the potential use of SuperDARN radars in mind and to test the theoretical predictions for dependence of the phase velocity of Farley-Buneman waves on radar frequencies in the HF range, a statistical analysis was made of over 11,000 specifically selected spectra from multi-frequency observations by the SuperDARN Pykkvibaer radar in September-October 2000. Good qualitative agreement was found between the observed and predicted frequency dependence for slightly disturbed magnetic conditions. Assuming that increased magnetic activity (higher $\mathrm{K}_{\mathrm{p}}$ ) manifests itself via enhanced electron temperature and applying the algorithm of the control parameters of Kagan \& Kissack [1], it was shown that in agreement with observations, the dependence of the FB waves phase velocity on the irregularity wave number (radar frequency) should decrease with increasing electron temperature $\left(\mathrm{K}_{\mathrm{p}}\right)$. The results make it clear that specially designed multi-frequency SuperDARN experiments would be a valuable tool in studying the HF FarleyBuneman waves at high latitudes.
\end{abstract}

Keywords: Farley-Buneman waves, thermal effects, high-latitude ionosphere, SuperDARN.

\section{INTRODUCTION}

The importance of two-stream processes in the formation of ionospheric irregularities has led to more and more complex linear and non-linear theories and increasingly sophisticated computer simulations of Farley-Buneman (FB), or two-stream, waves [2,3]. Non-linear theories [4-6] have been focusing on the mechanisms which produce the FB irregularities; computer simulations have been looking into saturation mechanisms for the FB waves growth [7-8] and their non-linear dynamics [9], while linear theories have been aimed at providing more refined expressions for the instability threshold speed as well as understanding the attendant physics.

Continuing strong interest in the linear approach is due to the remarkable property of the Farley-Buneman waves to travel with their phase speed of the order of the ion-acoustic speed $\mathrm{c}_{\mathrm{s}, \mathrm{i}}$ which, in standard classical theory, is the speed of waves moving at their linear instability threshold. The increased degree of sophistication in the linear fluid theories was at first limited to isothermal treatments, culminating in the generalization presented by Fejer et al. [10]. Gradually, thermal processes were also added to the calculations both in kinetic and, mostly, in fluid treatments of the problem. The addition of thermal effects led to the discovery of additional feedback mechanisms which had either a positive or a negative effect on threshold properties, depending on conditions (e.g., [11-24]).

Farley and Providakes [25] were the first to recognize that the threshold speed of small-scale E region irregularities should be evaluated more carefully, based on their

*Address correspondence to this author at the Department of Physics and Astronomy, University of Western Ontario, 1151 Richmond Street, London ON, N6A 3K7, Canada; Tel: 1-519-661-2111, Ext. 81101; Fax: 1-519-6612033; E-mail: 1kagan@uwo.ca observations of high latitude $\mathrm{E}$ region irregularities moving with phase speeds clearly faster than the isothermal ionacoustic speed, and much closer to a speed associated with adiabatic electrons. Following the work of Farley and Providakes [25] there have been two distinct approaches to this problem, both based on non-isothermal electron corrections. In the series of papers written by Dimant and Sudan, the focus was on an improved kinetic starting point for the instability calculations [15-18].

The other approach by the group to which this paper belongs [13-14, 21-24], was based on Grad's set of fluid equations closed at the heat flow level; it self-consistently describes the effects of collisions using Burgers' expressions for collision integrals [26]. This approach was shown to be very fruitful when, despite being limited to zero aspect and flow angles, the formulation by St.-Maurice and Kissack [21] allowed St.-Maurice et al. [27] to explain, for the first time, the puzzling fact that two-step type-I waves in the lower electrojet moved at speeds up to $50 \%$ higher than the isothermal ion acoustic speed. An advantage of this approach is that the contributions of thermal corrections can be written as additional terms to the isothermal theory.

J.-P. St.-Maurice was the first to realize that when the Farley-Buneman wave phase velocities were about 1.5 times higher than the isothermal ion-acoustic speed [27], they still matched remarkably well the non-isothermal ion acoustic velocity, therefore confirming the concept that FB waves move at a speed of their linear instability threshold, but this linear threshold is the non-isothermal ion acoustic speed [1, $13,14,21,27]$.

Furthermore, observations indicate that waves often move at the threshold speed even when the electric field is strong enough to excite waves that could be moving much faster than that. Several nonlinear theories and simulations have been proposed to explain this (see for example [4-8]). Investigating non-isothermal linear threshold $\mathrm{FB}$ velocities, 
Kagan and St.-Maurice [22] showed that although the threshold phase speeds would be greater in the strongly driven case of very high electric fields (triple that of the linear threshold), the numbers are not changing that much at lower altitudes or for aspect angles $>0.3^{\circ}$. They obtained the largest deviation from the linear threshold for the altitudes $>110 \mathrm{~km}$ and small enough aspect angles $\left(<0.1^{\circ}\right)$.

The next step exploring aspect sensitivity [22] showed good correspondence to observations by Kudeki and Farley [28] and good qualitative agreement of observed altitude behavior of Farley-Buneman waves being super-adiabatic at lower altitudes and isothermal at high altitudes.

The latest development of the theory [23,24], which includes non-zero flow angles and an arbitrary heat source (with possible applications for high latitudes and heating experiments) presented the results in such a way that allowed one to clearly identify contributions from each physical process and to easily compare the results with previous work. Applying this theory to the three-frequency observations of Balsley and Farley [29], Kagan and Kissack [1] showed that the frequency dependence predicted by the Kissack et al. [24] theory matched the data remarkably well. Kagan \& Kissack [1] also introduced a simplified expression for the phase velocity of Farley-Buneman waves, which facilitated quick estimates and also permitted the identification of the physical process that dominated behavior of FB waves at a given frequency and altitude.

Kagan et al. [30] further advanced applications of the Kissack et al. $[23,24]$ theory by introducing two control parameters $\xi_{A T}$ (for super-adiabatic) and $\xi_{T}$ (for thermal conduction/diffusion) which define behavior of the FB wave. This in turn, allowed them to explain an unexpected and drastic drop in the phase velocity, $V_{p h}$, of Farley-Buneman waves with increasing altitude observed in the equatorial electrojet over Jicamarca with the newly employed $430-\mathrm{MHz}$ radar looking vertically. In particular they showed that electron inelastic cooling, which defines a gradual transition from super-adiabatic to isothermal processes at $50 \mathrm{MHz}$ (most commonly used in observations), becomes unimportant at higher frequencies.

It is of particular interest to extend the comparison of theory and experiment into the HF radar range where comparisons have so far been very limited (only the equatorial electrojet observations by Balsley and Farley at 16 $\mathrm{MHz}$ during their multi-frequency experiment [29]). The best opportunities are in the auroral electrojet region, where a large number of SuperDARN radars are operating in this frequency range. However, because the vertical beam pattern of the SuperDARN radars is purposely very broad in order to receive echoes from both the E- and F-regions over a range spread of several thousand kilometers, there is no information on the height from which the echoes come. Though the simultaneous VHF and UHF multi-frequency observations of aurora performed in nineteen sixties [31] showed that radar echoes, presumably associated with the auroral electrojet, were coming from the 100-120 km altitudes, the wide altitude extent of $20 \mathrm{~km}$ would imply drastically different ionospheric parameters. Ruohoniemi and Moorcroft [32] and Moorcroft and Ruohoniemi [33] were able to get height resolutions of about $3 \mathrm{~km}$ using a careful analysis of data from the $398 \mathrm{MHz}$ Homer radar and found the similar altitude coverage, the echo heights varied from as low as $98 \mathrm{~km}$ to as high as $115 \mathrm{~km}$. Therefore the only way to test the validity of our theory for HF SuperDARN radars, would be to make use of multi-frequency observations, as was previously done with the Balsley and Farley [29] data.

Note that this work is limited to the HF high-latitude observations only (with tentative applications to the SuperDARN data), for a summary of VHF and UHF auroral studies we refer the reader to the excellent review by Sahr and Fejer [34] and the references therein.

The paper starts with a theoretical description followed by observations. The latter includes the data-selection procedure, data, discussion of flow angle and frequency dependences of FB phase velocities, $V_{\mathrm{ph}}$, and a $V_{\mathrm{ph}}$ dependence on geomagnetic activity. The discussion of the results and conclusion are given in the last section.

\section{THEORETICAL DESCRIPTION}

As shown by Kagan and Kissack [1] and Kagan et al. [30], the threshold phase velocity of Farley-Buneman waves depends largely on three different parameters: (1) the magnetic aspect angle, $\phi$ (angle between the wave vector $\mathbf{k}$ and the plane perpendicular to the background magnetic field); (2) the flow angle, $\theta_{f}$ (angle between $\mathbf{k}$ and the relative ion-electron drift $\mathbf{u}$ ); and (3) the height of the backscatter, through the height dependence of collisional frequencies, electron temperature, and the inelastic electronneutral energy exchange rate. These three dependences each present problems when working with SuperDARN data, since neither the backscatter height nor the magnetic aspect angle are known, and there is very limited control over the flow angle.

We are going to deal with these problems in various ways. First, we will assume that the magnetic aspect angle $\phi$

is zero. This is a reasonable assumption since the backscatter echo strength is expected to be a maximum when $\phi=0$, and at HF there is significant refraction which makes the condition of zero aspect angle easily attained much of the time. Moreover, all careful studies of magnetic aspect effects have shown that the backscatter power falls off very rapidly with increasing aspect angle. As a consequence, the majority of observed backscatter comes from close to zero aspect angle, and in a statistical study, the behavior will be dominated by that majority of strong, essentially zero aspect angle echoes.

Second, we will also assume that the flow angle is zero, and will justify that assumption by appropriate selection of the experimental data, supported by evidence in the data which is consistent with the assumption. We will deal with the problem of the unknown height by presenting theoretical results as functions of the height for each radar frequency and comparing the difference between the phase velocities at different frequencies.

Our starting point is the expression for the phase velocity of Farley-Buneman waves under conditions of marginal stability, introducing the assumptions of zero aspect and 
flow angles as we go. The following expression is slightly different in form but identical in content to that given by Kagan and Kissack [1] which is based on much more complex formulas given by Kissack et al. [23,24]: magnitudes indicate which processes are dominant in $V_{\mathrm{ph}}$. To obtain the following expressions from those in Kagan and Kissack we have taken $\phi$ to be zero, expanded intermediate quantities, and used the fact that the thermal corrections to

$$
V_{p h}=u_{i 0} \pm c_{s, i} \frac{k_{\perp}}{k} \sqrt{1+\frac{T_{e 0}}{\left(T_{e 0}+T_{i 0}\right)} \frac{\chi^{D S}(2 / 3) \eta_{e}\left[C D_{e \perp} k_{\perp}^{2}+\eta_{e}\right]+(1+g)^{2} A^{2}\left[\psi_{T} /\left(1+\psi_{T}\right)\right]^{2} u_{0}^{2} k^{2}}{\left\{(3 / 2)\left[\psi_{T} /\left(1+\psi_{T}\right)\right]^{2} u_{0}^{2} k^{2}+(2 / 3)\left[C D_{e \perp} k_{\perp}^{2}+\eta_{e}\right]^{2}\right\}}}
$$

where $V_{\mathrm{ph}}$ is the phase speed with an account for the ion drift velocity, $u_{\mathrm{i} 0}$, in the line of sight of the radar. In the equation $c_{s, i}=\sqrt{k_{T}\left(T_{e 0}+T_{i 0}\right) / m_{i}}$ is the isothermal acoustic speed; $\kappa_{T}$ is Boltzmann constant, $T_{\mathrm{e} 0}$ and $T_{\mathrm{i} 0}$ are the background electron and ion temperatures, respectively, and $m_{\mathrm{i}}$ is the mean ion mass; $k$ is the magnitude of the wave vector and $k_{\perp}$ is the magnitude of the wave vector component perpendicular to the geomagnetic field (equal to $k$ for $\phi=0$ ).

$$
\begin{gathered}
A\left(\frac{k_{\|}^{2}}{k_{\perp}^{2}}\right)=\left[1+\frac{(\alpha-g)}{(1+g)} \beta\right] /[1+\alpha \beta] \\
C\left(\frac{k_{\|}^{2}}{k_{\perp}^{2}}\right)=5 / 2+2 g+g^{2}-(g-5 / 2) \beta- \\
g(1+g)(1-\beta) A\left(\frac{k_{\|}^{2}}{k_{\perp}^{2}}\right) \\
g=\left[\frac{T_{e 0}}{v_{e}} \frac{\partial v_{e}}{\partial T_{e}}\right]_{T_{e 0}}, \alpha=1+2 g(1+g) / 5, \\
\beta=\frac{1}{(1+2 g / 5)} \frac{k_{\|}^{2} \omega_{e}^{2}}{k_{\perp}^{2} v_{e n}^{2}} . \\
\psi_{T}=\psi_{0}(1+\alpha \beta), \psi_{0}=\frac{v_{e} v_{i n}}{\omega_{e} \omega_{i}}, \eta_{e}=\delta_{e} v_{e}, \\
\chi^{D S}=\left[(1+g)^{2} A-3 g \bar{s} / 2\right] \bar{s}
\end{gathered}
$$

is the term describing Dimant-Sudan instability where the parameter

$$
\bar{s}=\frac{k_{\perp}^{2} u_{0}^{2} \sin \theta_{f} \cos \theta_{f}}{D_{e \perp} k^{2} \eta} \frac{v_{e}}{\omega_{e}} \frac{\psi_{0}}{\left(1+\psi_{T}\right)}
$$

is a function of a flow angle $\theta_{f}$ resulting in zeroing the Dimant-Sudan instability effects at $0^{\circ}$ and $90^{\circ}$ flow angles; $v_{e}, v_{i}, \omega_{e}, \omega_{i}$ are the electron and ion collisional and cyclotron frequencies, respectively; $\eta_{e}=\delta_{e} v_{e}$ is the inelastic volume electron-neutral energy exchange rate, $\delta_{e}$ is a dimensionless energy exchange factor, and $m_{\mathrm{e}}$ is the electron mass.

The two quantities $\xi_{T}=C D_{e \perp} k_{\perp}^{2} / \eta_{e} \quad$ and $\xi_{A T}=3\left[\psi_{T} /\left(1+\psi_{T}\right)\right]^{2} u_{0}^{2} k^{2} / \eta_{e}$ are dimensionless control parameters introduced by Kagan and Kissack [1], whose the frequency of Farley-Buneman waves are less than 3\% in most cases [22] to write $\omega_{r}-\mathbf{k} \cdot \mathbf{u}_{0} \approx \omega_{r}-\left(1+\psi_{T}\right) \omega_{r}=\psi_{T} \omega_{r}$, which for $\phi=0$ equals $\psi_{0} \omega_{r}$, where $\mathbf{u}_{0}=\mathbf{V}_{e 0}-\mathbf{V}_{i 0}$ is the relative velocity between ions and electrons. The result is

$$
\begin{aligned}
& \xi_{A T}=\frac{3 \psi_{0}}{2 \eta_{e}} V_{p h} k=\frac{\text { Inertial effects }}{\text { Collisional energy exchange }}, \\
& \xi_{T}=\frac{10 v_{e} \kappa_{T} T_{e 0}}{3 \eta_{e} m_{e} \omega_{e}^{2}} k^{2}=\frac{\text { Thermal conduction }}{\text { Collisional energy exchange }} .
\end{aligned}
$$

Rewriting (1) in terms of the control parameters gives

$$
V_{p h}=u_{i 0} \pm c_{s, i} \frac{k_{\perp}}{k} \sqrt{1+\frac{T_{e 0}}{\left(T_{e 0}+T_{i 0}\right)} \frac{\chi^{D S}\left(1+\xi_{T}\right)+\chi^{A T} \xi_{A T}^{2}}{\left\{\xi_{A T}^{2}+\left(1+\xi_{T}\right)^{2}\right\}}}
$$

where the quantity $\chi^{A T}=2(1+g)^{2} A^{2} / 3$ depends on contributions from adiabaticity (including thermal corrections), and is a strong function of the magnetic aspect angle $\phi$. If $\phi=0$ then, assuming as accustomed $g=5 / 6, \chi^{A T}$ reduces to a constant $\chi^{A T} \approx 2.24$.

Finally, $\chi^{D S}$ (Eqs. 6 and 7) is an expression giving the contribution to $V_{\mathrm{ph}}$ from the Dimant-Sudan effects (see [24] for details). It depends on the flow angle and is zero if the flow angle is either $0^{\circ}$ or $90^{\circ}$. At HF radar frequencies the Dimant-Sudan effects can be quite important, significantly modifying the frequency dependence of the threshold phase velocity. But our choice of data allowing the assumption of a zero flow angle eliminates this term.

With the assumptions of a zero flow angle and a zero magnetic aspect angle, the expression for the threshold phase velocity (10) simplifies considerably. Furthermore, when substituting some typical values for the various quantities into the expression for $\xi_{T}$ (9), we find that for HF radar wavelengths $\xi_{T}$ is on the order of 0.01 . So to a good approximation $\left(\xi_{T}+1\right)^{2} \approx 1$. Therefore, similar to the result for $16 \mathrm{MHz}$ at Jicamarca and unlike the results for $50-\mathrm{MHz}$ and higher (see figure 4 in [1]), for HF radar frequencies the parameter $\xi_{T}$ does not play any role. The resulting expression for the phase velocity in the ion frame of reference is

$$
V_{p h} \approx c_{s, i} \sqrt{1+\frac{T_{e 0}}{\left(T_{e 0}+T_{i 0}\right)} 2.24\left(\frac{\xi_{A T}^{2}}{1+\xi_{A T}^{2}}\right)} .
$$


From equation (11) the frequency dependence of $V_{\mathrm{ph}}$ can be seen to depend entirely on the radar frequency and altitude (the last one via parameters $\psi_{0}$ and $\eta_{e}$ ):

$$
\xi_{A T}=\frac{3 \psi_{0}}{2 \eta_{e}} V_{p h}(2 \pi / c) f_{R}
$$

where $c$ is the speed of light and $f_{R}$ is the radar frequency.

If $\xi_{A T} \ll 1$ then the equation reduces to $V_{p h}=c_{s, i}$ and the phase speed is then independent of radar frequency.

If $\xi_{A T} \gg 1$ the phase speed is once again independent of radar frequency but now being super-adiabatic: $V_{p h}=c_{s, i} \sqrt{1+2.24 T_{e 0} /\left(T_{e 0}+T_{i 0}\right)}$. Only when $\xi_{A T} \sim 1$ is $V_{\mathrm{ph}}$ predicted by equation (4) to increase with increasing frequency.

For a given radar frequency change we can easily get an estimate of the corresponding change in phase velocity by using equation (11) to estimate $d V_{p h} / d f_{R}$ taking into account that $V_{\mathrm{ph}}$ depends on $f_{\mathrm{R}}$ parametrically through the $f_{\mathrm{R}}$ dependence of $\xi_{A T}$ as described by Eq. (12) where the radar wave number was substituted by the respective expression of the radar frequency. The result is

$$
\frac{\partial V_{p h}}{V_{p h}}=\frac{2.24 T_{e 0} /\left(T_{e 0}+T_{i 0}\right)}{\left[\left(1 / \xi_{A T}+\xi_{A T}\right)^{2} V_{p h}^{2} / c_{s, i}^{2}-2.24 T_{e 0} /\left(T_{e 0}+T_{i 0}\right)\right]} \frac{\partial f_{R}}{f_{R}} .
$$

The maximum difference of phase velocities at different HF radar frequencies would be when $\left(1 / \xi_{A T}+\xi_{A T}\right)$ is minimum, that is at $\xi_{A T}=1$, and $V_{p h} \approx c_{s, i}$, we arrive at

$$
\Delta V_{p h}^{\max } \approx 0.4 c_{s, i} \Delta f_{R} / f_{R} .
$$

We can use equation (14) to get an estimate of the magnitude of the frequency dependence effects to be expected for the experiments reported on in this paper. For these data $f_{R} \approx 13.24 \mathrm{MHz}$ with differences to the two neighboring frequencies $1.93 \mathrm{MHz}$ and $1.39 \mathrm{MHz}$ gives $\Delta f_{R} / f_{R} \approx 0.171$ and 0.105 . Since the data don't give us an altitude from which the radar echoes come, we may assume $c_{s, i} \approx 320-390 \mathrm{~m} / \mathrm{s}$ (100 to $110-\mathrm{km}$ altitude range) and obtain that for quiet conditions depending on altitude the expected maximum difference between the phase velocities of FB waves would be $14-27 \mathrm{~m} / \mathrm{s}$. This estimate is, of course, very approximate. So if we, for example, assume that the echoes come from $120 \mathrm{~km}$ altitude, then $c_{s, i}$ would be much higher, $471 \mathrm{~m} / \mathrm{s}$, which in turn would give the maximum velocity difference $\Delta V_{p h} \approx 32 \mathrm{~m} / \mathrm{s}$. Introduction of even small nonzero flow and aspect angles could further alter our estimate and would greatly increase the complexity of this simple calculation, possibly to the point of impracticality.

Note that the FB phase velocities would differ only when the controlling parameter $\xi_{A T} \sim 1$. For $\xi_{A T}<0.1$ or $>10 \Delta V_{p h}$ would become negligible. Clearly for HF Farley-Buneman waves the most important parameter defining frequency dependence of the FB phase velocity is $\xi_{A T}$.

If one were to assume that increased electron temperature goes with enhanced magnetic activity (higher $\mathrm{K}_{\mathrm{P}}$ indexes in our data), then the control parameter $\xi_{A T}$ would increase as well (via $\eta_{e}$ temperature dependence) from being $\sim 1$ to becoming increasingly higher than 1 . Therefore one could expect that the higher the magnetic activity, the smaller the difference between the FB phase velocities at different frequencies.

\section{DATA SELECTION}

The normal operating mode of SuperDARN radars does not include observations at multiple frequencies, but from time to time special programs are run which make use of multiple frequencies. In particular, a multiple frequency program (\#6007) was operated on 11 consecutive days on the Pykkvibaer radar between 22 September, 2000 and 02 October, 2000. The features of the program are shown in Table 1 below.

It was found that the usable data in the $9 \mathrm{MHz}$ band were too few to obtain any useful results, so this frequency band was not used in the present study. The SuperDARN experimental program which generated these data was designed for a different purpose, and that is why the frequencies are variable rather than fixed, and why the observations are restricted to daytime hours, neither choice being ideal for studying the frequency dependence of backscatter from Farley-Buneman instabilities.

Table 1. A Multiple Frequency Program \#6007, the Pykkvibaer Radar, 22/09-2/10/ 2000

\begin{tabular}{|c|c|}
\hline Hours: & $6: 00 \mathrm{UT}-18: 00 \mathrm{UT}$ daily \\
\hline Ranges: & $60 \mathrm{~km}-1170 \mathrm{~km}$, at $15 \mathrm{~km}$ intervals \\
\hline Frequen-cies: & $\begin{array}{c}\text { variable in four bands, roughly centered } \\
\text { on } 9,12,13 \text { and } 14.5 \mathrm{MHz}\end{array}$ \\
\hline Operation: & $\begin{array}{c}\text { Cycle through the } 4 \text { frequency bands, 34.33s for each } \\
\text { frequency, frequencies varying within each band } \\
\text { from cycle to cycle. }\end{array}$ \\
\hline
\end{tabular}

The SuperDARN radars use a multi-pulse scheme to achieve their range resolution. When echoes are spread in range, this can result in multi-pulse clutter - strong echoes producing significant returns in adjacent range gates. As a consequence of this issue, a significant fraction of the observed spectra are very irregular, with multiple peaks and no easily identified ion-acoustic peak.

The routine SuperDARN analysis procedures were not used; instead the spectra were calculated directly from the raw autocorrelation function data. In studies with SuperDARN and other radars it has been found that a simple exponential power law does a good job of fitting the autocorrelation functions associated with clean spectra [35], so this approach has been used here to reject spectra contaminated by multi-pulse clutter, leaving a database consisting of only single-peaked, relatively smooth spectra. 
The vertical beam pattern of the SuperDARN radars is purposely very broad in order to receive echoes from both the E- and F-regions over a wide spread of ranges. Thus, there is no information on the height from which the echoes come. The SuperDARN radars actually do have an interferometer capability which can, in principle, provide some elevation angle information, but in practice it is unreliable, and in particular the Pykkvibaer interferometer is unable to add useful information on what part of the Eregion the echoes are coming from.

Because of the lack of height information, there is no way to ensure that the echoes are coming only from the Eregion. At the nearest ranges that is not an issue, but beyond a few hundred kilometers there may be a significant fraction of F-region echoes as well. For that and other reasons it is not possible to arrange to view only ion-acoustic echoes from the E-region. We could expect to often see FarleyBuneman echoes superimposed on top of a background of echoes of other types.

Ideally, we would have virtually simultaneous echoes on all three frequencies from the same range gates. In practice, after filtering for suitable spectra, it was found that such simultaneous, co-located multi-frequency echoes are very rarely observed. Consequently, the only feasible approach is to do a statistical analysis, in the hope that the average behavior of the ion-acoustic spectra would provide a suitable basis for comparison with theory. This approach is also appropriate for dealing with the problems of the unknown aspect angle, flow angle and E-region height, as mentioned in the last section.

It is expected that the easiest observations to interpret will be those obtained during relatively quiet conditions. Also as we mentioned in Theoretical Description above, the difference between phase velocities of FB waves at different HF frequencies will be larger, disappearing with increased magnetic activity. For this reason, the $K_{p}$ index corresponding to each selected spectrum has been recorded.

We have also estimated the flow angle corresponding to each spectrum by recording the L-shell direction at the observation field point. For any given observation it is unlikely that the current direction will be exactly along the L-shell, but in a statistical analysis, it is hoped that the flow direction can be sufficiently well constrained to allow meaningful results to be obtained.

Since the purpose of this study is to look at the frequency dependence of the Farley-Buneman phase speeds (radar Doppler speeds), it is an inconvenience of this data set that in each frequency band the radar frequency changes somewhat randomly over each of those bands. It has been found that a judicious selection of maximum and minimum frequencies in each band could keep a large fraction of the data while minimizing the frequency spread within each band. The resultant frequency ranges analyzed were $11.29-11.33$ $\mathrm{MHz}, 13.20$ - $13.279 \mathrm{MHz}$, and 14.58 - 14.68 MHz.

In summary, the analysis proceeded as follows: for each of the 11 days during which program \#6007 was run in September-October 2000, all smooth, single peak spectra corresponding to radar frequencies within the ranges mentioned in the last paragraph were selected, and the time, $\mathrm{K}_{\mathrm{p}}$ value, estimated flow angle, and speed (in $\mathrm{m} / \mathrm{s}$ ) corresponding to the peak of each spectrum were recorded.

Since we are interested in Farley-Buneman waves, these were further culled to eliminate all those values for which the speed of the spectral peak was less than $200 \mathrm{~m} / \mathrm{s}$. This eliminated a large fraction of the spectra, and resulted in a database of slightly more than 11,000 records.

\section{OBSERVATIONS}

Although data were available for four 3-hour periods (corresponding to $\mathrm{K}_{\mathrm{p}}$ intervals) on each of the 11 days of the experiment, ion-acoustic spectral peaks were observed only during the periods after $12 \mathrm{UT}$, with most of them occurring between 15 and 18 UT. Of those remaining twenty two 3hour periods, only 7 three-hour periods, spanning a range of $\mathrm{K}_{\mathrm{p}}$ values from 2- to 5 , have been used in the analysis, as indicated in Table 2. Although some data were obtained in other 3-hour periods for some of these $\mathrm{K}_{\mathrm{p}}$ values, they were significantly fewer in number than for the periods in the table, and it was considered better to constrain the time period for the data as much as possible, by keeping only those periods during which there was a significant amount of activity.

In Table 2, all the three-hour periods were from 15 to 18 UT, with the exception of the $\mathrm{K}_{\mathrm{p}}=3+$ data which occurred between 12 to 15 UT.

The map in Fig. (1) shows the field of view of the Pykkvibaer radar, as well as the distributions of the used echoes in range and beam number. The ranges are concentrated in the $400-700 \mathrm{~km}$ range interval, with a tail to greater ranges. The weighting of the beams towards the south is entirely reasonable for echoes coming from the afternoon (12 - 18 UT), since then the most favorable flow angles would be expected to lie just southward of the most southerly beam.

Table 2. Numbers of Data and Dates for Various $K_{p}$ Values

\begin{tabular}{|c|c|c|c|c|c|c|c|c|c|}
\hline $\mathbf{K}_{\mathbf{p}}$ & Frequency & $2-$ & 3- & 3 & $3+$ & 4 & $4+$ & 5 & Totals \\
\hline \multirow[t]{2}{*}{ Number of Data } & $13 \mathrm{MHz}$ & 867 & 506 & 530 & 257 & 625 & 490 & 519 & 3794 \\
\hline & $14 \mathrm{MHz}$ & 404 & 925 & 444 & 313 & 716 & 433 & 383 & 3628 \\
\hline
\end{tabular}




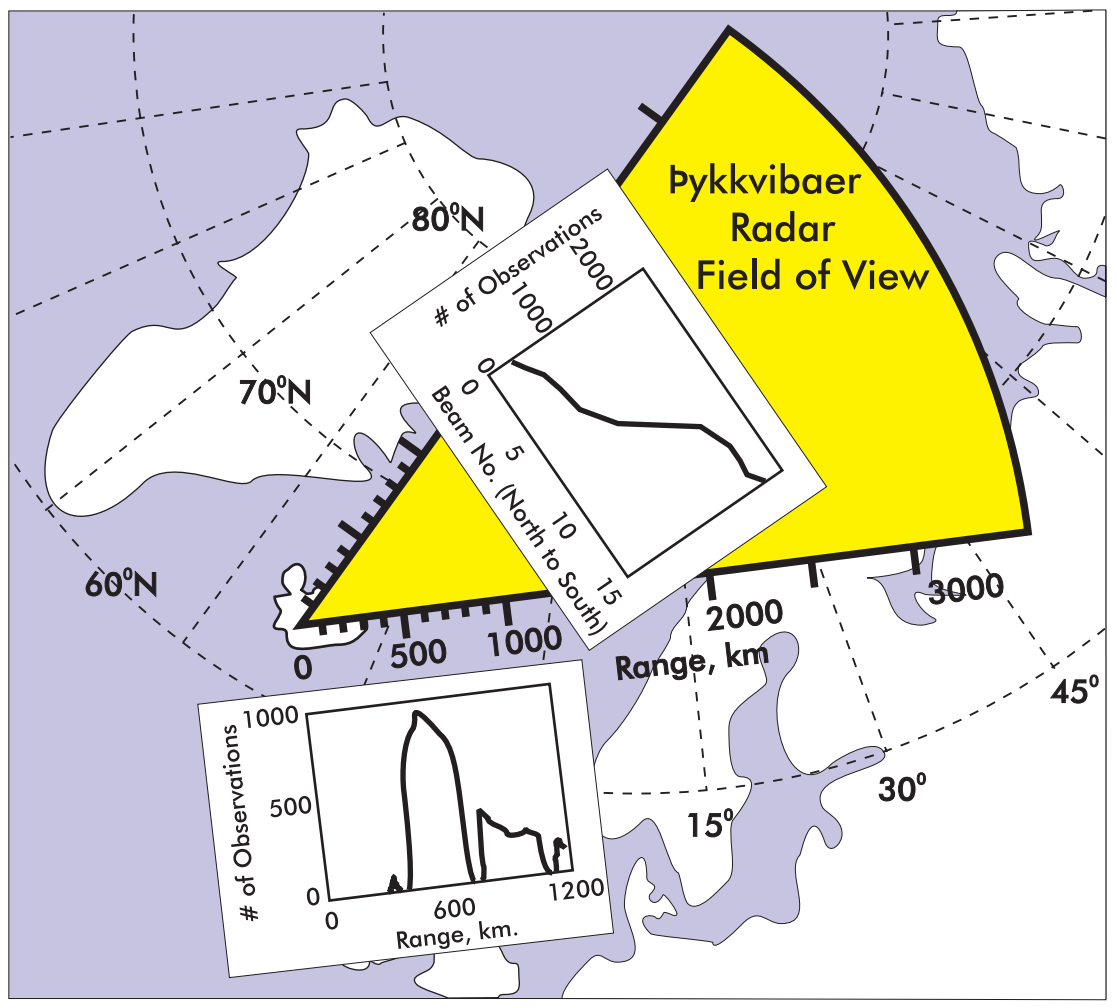

Fig. (1). The geographic location of the Pykkvibaer radar's field of view. Also shown superimposed on the map are the distributions of the used echoes in range and beam number.

\subsection{Flow Angle Dependence}

As mentioned earlier, we have used L-shell directions as a way of approximately ordering data by flow angles, while recognizing that the true flow angle can vary significantly from these L-shell directions. Fig. (2) shows the distributions of peak radar Doppler speeds for $\mathrm{K}_{\mathrm{p}}=3$ - and for L-shell angles (angle between radar line-of-sight and the L-shell at the field point) in four different ranges: $10^{\circ}-20^{\circ}, 15^{\circ}-25^{\circ}$, $20^{\circ}-30^{\circ}$, and $30^{\circ}-60^{\circ}$, covering the total range of possible Lshell angles for the radar at these times of day.

These flow-angle ranges are as small as possible while still maintaining sufficient numbers of data to provide reasonable distributions. What is evident from these plots is that the distributions get progressively broader with increasing L-shell angle. We have estimated the widths of these distributions for all seven values of $K_{p}$ and for all three radar frequency ranges, and have averaged the widths for each of five ranges of L-shell angles (the four used in Fig. (1) plus $25^{\circ}-35^{\circ}$, as shown in Fig. (3)).

On average there is a very little difference between the distribution widths up to an L-shell angle of $25^{\circ}$, but for larger angles the distributions become increasingly spread. We interpret this result as indicating that below an L-shell angle of $25^{\circ}$ there are no discernible flow angle effects on the observed phase velocities. At larger L-shell angles the increased spread in velocities likely results from the growing importance of flow angle effects.

\subsection{Frequency Dependence of Phase Velocities}

From Fig. (2) it can be seen that in three of the four panels the peak of the distribution moves to successively higher velocity as the radar frequency increases, in agreement with the predictions of the theory in Section 2. In Fig. (4) are shown the distributions for the other six levels of $\mathrm{K}_{\mathrm{p}}$ for the L-shell ranges of $10^{\circ}-20^{\circ}$ and $20^{\circ}-30^{\circ}$, which have been chosen since they are comprise of completely independent sets of data. Several points can be made about these data, along with those of Fig. (2): (1) the data for $K_{p}=$ 3 are quite anomalous, with unusually high velocities, and no consistent frequency dependence (since these data are from the same time period on September 26, $V_{\text {ph }}$ were most probably affected by an unusually strong neutral wind or/and electric field); therefore we will make no further use of these data; (2) the remaining 6 plots for $K_{p}$ less than 4 all show a consistent increase in velocity with frequency, while the plots for $\mathrm{K}_{\mathrm{p}} \geq 4$ are more muddled, with only two of the six plots showing a steady progression peak location with frequency; (3) the magnitudes of the velocity differences from one frequency to another are quite variable.

In order to reduced somewhat the variability of the velocity differences, in Table $\mathbf{3}$ below we give the velocity differences $\quad \Delta V_{12}=V_{13 \mathrm{MHz}}^{\text {peak }}-V_{11 \mathrm{MHz} z}^{\text {peak }}$ and $\Delta V_{23}=V_{14 \mathrm{MHz}}^{\text {peak }}-V_{13 \mathrm{MHz}}^{\text {peak }}$, averaged over values of $K_{p}<4$ and $\geq 4$, for three different ranges of L-shell angle. 

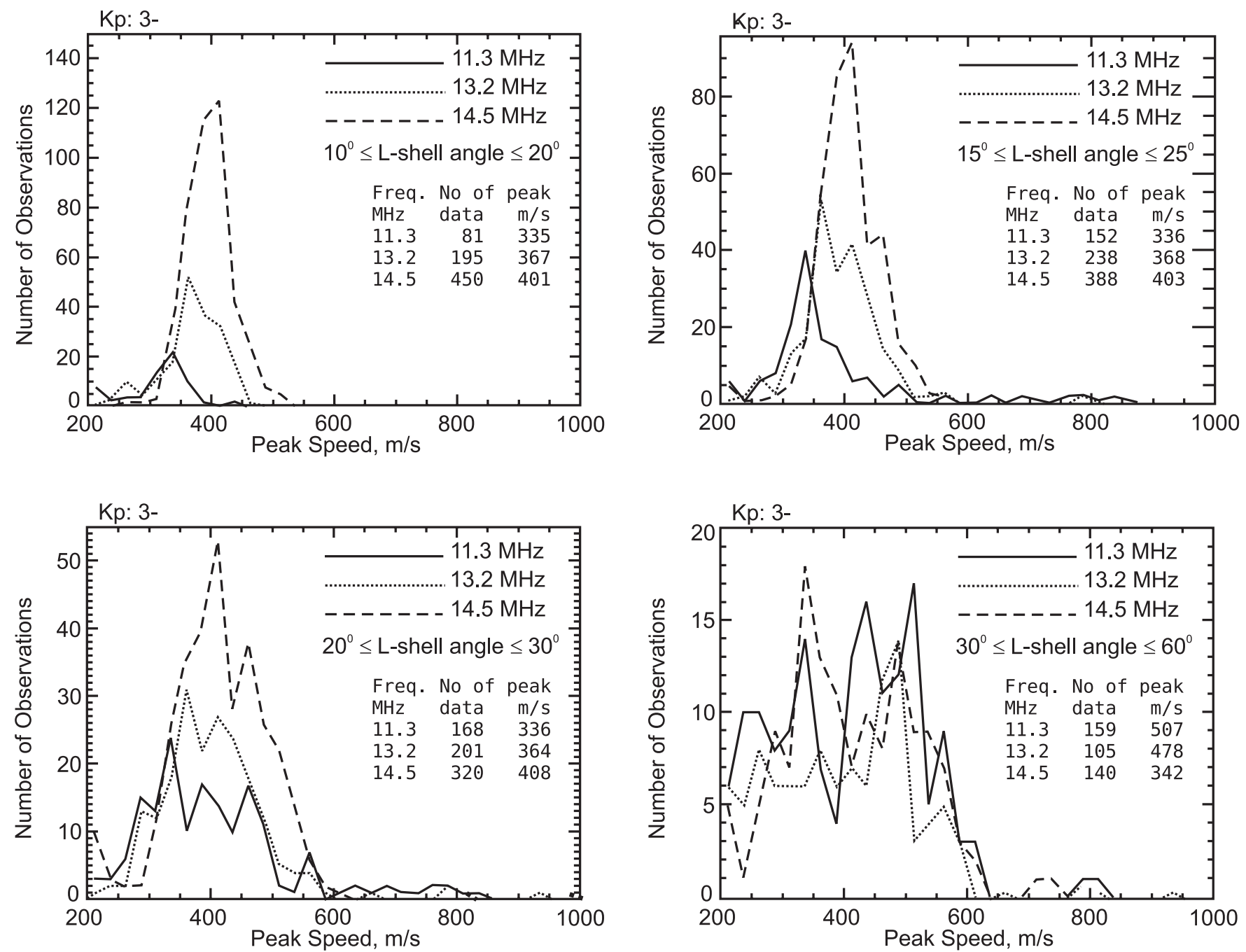

Fig. (2). Distributions of spectral peaks: $K_{p}=3$ - and various L-shell directions.

The average values of $\Delta V_{12}$ and $\Delta V_{23}$ for the lower values of $\mathrm{K}_{\mathrm{p}}$ are remarkably consistent from one L-shell angle range to the next, and agree reasonably well with the predictions of equation (14).

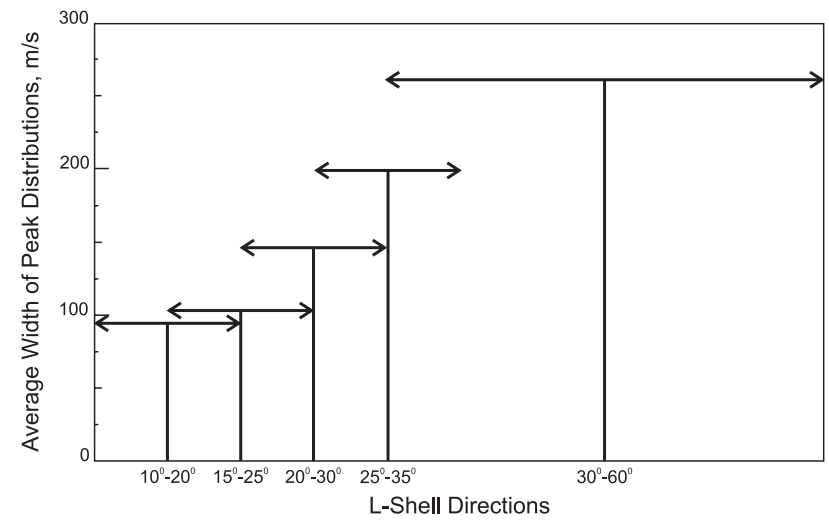

Fig. (3). Widths of distributions of peak velocity for different ranges of L-shell direction, averaged over all radar frequencies and $\mathrm{K}_{\mathrm{p}}$ values

It is not a great surprise that the data from the higher levels of $\mathrm{K}_{\mathrm{p}}$ are so disordered. It is well known that these very disturbed conditions are characterized by large and rapid variations, so averaging data from such a three-hour period would reflect the turbulent conditions prevailing during that time. Note, that differences between $V_{\mathrm{ph}}$ at different frequencies decrease with increasing magnetic activity, very much in agreement with our theoretical predictions in Section 2. We explain this by the change in the control parameter $\xi_{A T}$ increasing from $\sim 1$ to values $>1$ and therefore by shifting the dominating regime from intermediate, defined by electron energy exchange (as defined by Kagan and Kissack [1]), to super-adiabatic with increasing electron temperature.

\section{DISCUSSION AND CONCLUSIONS}

To extend the comparison of theory and experiment for Farley-Buneman waves into the HF radar range and with the potential use for SuperDARN radars in mind, we have studied data from the SuperDARN Dykkvibaer radar operating in the auroral electrojet region. Because the SuperDARN radars are designed to receive echoes from both the E- and F-regions and therefore don't provide information on the height from which the echoes come we have focused our analysis on multi-frequency observations. A special 

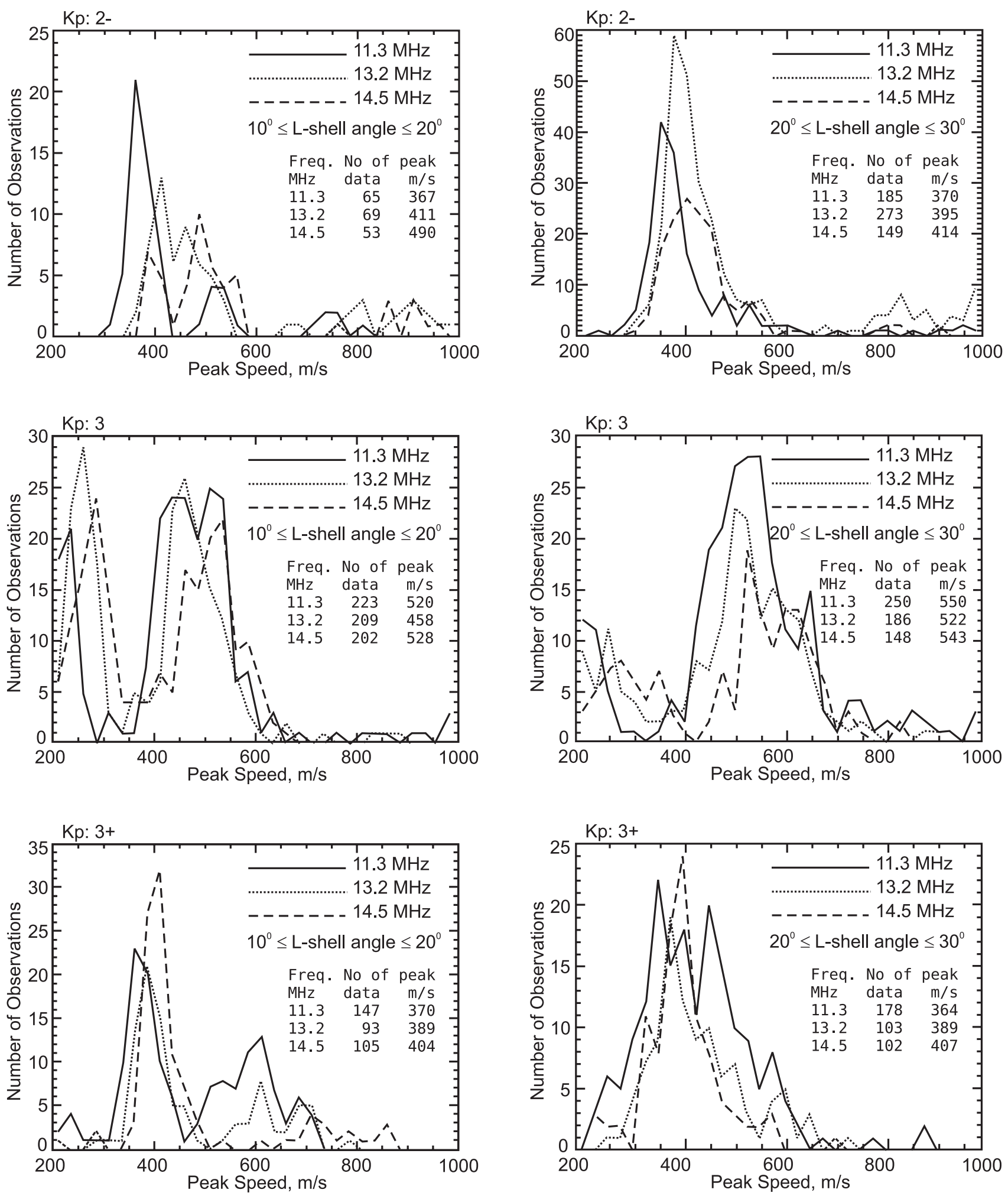

Fig. (4a). Distributions of spectral peaks for $\mathrm{K}_{\mathrm{p}}=2-, \mathrm{z}^{2}, 3+$ and L-shell angles $10^{\circ}-20^{\circ}$ and $20^{\circ}-30^{\circ}$.

program (\#6007) suitable for our purposes was run in September-October 2000.

In order to manage the problems related to the specifics of the SuperDARN observations, such as different range gates from which the radar returns come at different frequencies and unknown aspect and flow angles, we have done a statistical analysis, in the hope that the average behavior of the ion-acoustic spectra would provide a suitable basis for comparison with theory. To deal with a change in ionospheric conditions (parameters) associated with 

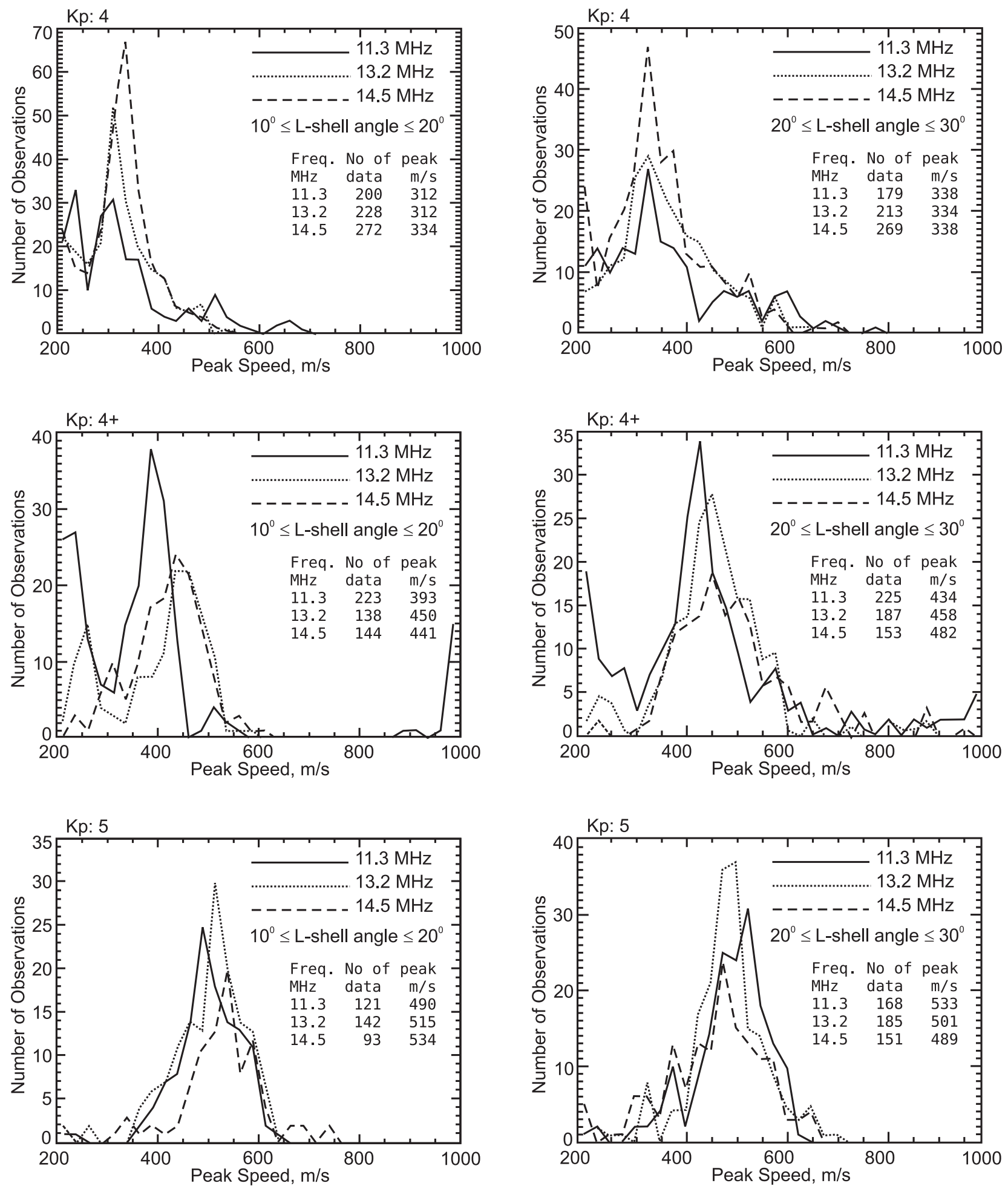

Fig. (4b). Distributions of spectral peaks for $\mathrm{K}_{\mathrm{p}}=4,4+, 5$ and L-shell angles $10^{\circ}-20^{\circ}$ and $20^{\circ}-30^{\circ}$.

enhanced magnetic activity, each selected spectrum has been recorded with its corresponding $\mathrm{K}_{\mathrm{p}}$ index.

The statistical approach allowed us to limit our analysis to zero aspect and flow angles and minimize frequency spread in each of the three frequency bands (11.29 - 11.33 $\mathrm{MHz}, 13.20$ - $13.279 \mathrm{MHz}$, and 14.58 - $14.68 \mathrm{MHz})$ selected. After limiting the data selection to all smooth, single peak spectra and eliminating the spectra with peak velocity less than $200 \mathrm{~m} / \mathrm{s}$ (since we are interested in FarleyBuneman waves) we have ended up with a database of 11,594 records. 
Table 3. The Velocity Differences $\Delta V_{12}$ and $\Delta V_{23}$, Averaged Over Values of $K_{p}<4$ and $\geq 4$, for Three Different Ranges of L-Shell Angle

\begin{tabular}{|c|c|c|c|c|c|c|}
\hline \multirow{2}{*}{ L-Shell Angles } & \multicolumn{2}{|c|}{$\mathbf{1 0}^{\circ}-\mathbf{2 0}$} & \multicolumn{2}{c|}{$\mathbf{1 5}^{\circ}-\mathbf{2 5}{ }^{\circ}$} & \multicolumn{2}{c|}{$\mathbf{0}^{\circ} \mathbf{3 0}^{\circ}$} \\
\hline \hline $\mathrm{V}_{\mathrm{ph}}$ difference & $\Delta \boldsymbol{V}_{\mathbf{1 2}}$ & $\Delta \boldsymbol{V}_{\mathbf{2 3}}$ & $\Delta \boldsymbol{V}_{\mathbf{1 2}}$ & $\Delta \boldsymbol{V}_{23}$ & $\Delta \boldsymbol{V}_{\mathbf{1 2}}$ & $\Delta \boldsymbol{V}_{\mathbf{2 3}}$ \\
\hline $\mathrm{K}_{\mathrm{p}}=2-, 3-, 3+$ & $32 \mathrm{~m} / \mathrm{s}$ & $43 \mathrm{~m} / \mathrm{s}$ & $34 \mathrm{~m} / \mathrm{s}$ & $38 \mathrm{~m} / \mathrm{s}$ & $26 \mathrm{~m} / \mathrm{s}$ & $27 \mathrm{~m} / \mathrm{s}$ \\
\hline $\mathrm{K}_{\mathrm{p}}=4,4+, 5$ & $27 \mathrm{~m} / \mathrm{s}$ & $11 \mathrm{~m} / \mathrm{s}$ & $-5 \mathrm{~m} / \mathrm{s}$ & $24 \mathrm{~m} / \mathrm{s}$ & $-4 \mathrm{~m} / \mathrm{s}$ & $-1 \mathrm{~m} / \mathrm{s}$ \\
\hline
\end{tabular}

Our statistical analysis of multi-frequency observations by the SuperDARN bykkvibaer radar have showed a good qualitative agreement between the observed frequency dependence for slightly disturbed magnetic conditions and the one predicted by the Kagan and Kissack [1] and Kissack et al. $[23,24]$ theories. Assuming that increased magnetic activity (higher $\mathrm{K}_{\mathrm{p}}$ ) manifests itself via enhanced electron temperature and applying the algorithm of the control parameters developed in [1], we have shown that in agreement with observations, the dependence of the FB waves phase velocity on the irregularity wave number (radar frequency) decreases with increasing electron temperature $\left(\mathrm{K}_{\mathrm{p}}\right)$.

Starting from $\mathrm{K}_{\mathrm{p}}=3$ and higher some other factors in addition to an increased electron temperature seem to contribute to the Farley-Buneman wave phase speed. This case clearly needs special consideration to explain the very peculiar enhancement in the phase speed of Farley-Buneman waves at $\mathrm{K}_{\mathrm{p}}=3$ and $\mathrm{K}_{\mathrm{p}}=4+$.

Perhaps the greatest surprise of this study is that a positive result of any kind could come from such a statistical analysis, depending as it does on so many assumptions, and based on data from an experiment which was far from ideal for the purposes to which it has been put. It is clear though that specially designed multi-frequency SuperDARN experiments would be a valuable tool in studying the HF Farley-Buneman waves at high latitudes both on their own and to complement observations of aurora by other means.

\section{ACKNOWLEDGEMENTS}

This research was supported by a grant from the Natural Sciences and Engineering Research Council of Canada. LMK's work has been supported by The Canadian Space Agency's ePOP/Cassiope mission program.

\section{REFERENCES}

[1] Kagan LM, Kissack RS. Energy exchange rate for the equatorial electrojet: test of the model of two-stream processes that includes thermal corrections. Geophys. Res Lett 2007; 34: L20806.

[2] Farley DT. A plasma instability resulting in field-aligned irregularities in the ionosphere. J Geophys Res 1963; 68: 6083-97.

[3] Buneman O. Excitation of field-aligned sound waves by electron streams. Phys Rev Lett 1963; 10: 285-7.

[4] Robinson TR. Towards a self-consistent nonlinear theory of radar auroral backscatter. J Atmos Terr Phys 1986; 48: 417-23.

[5] Hamza A, St-Maurice J-P. Large aspect angles in auroral E region echoes: a self-consistent turbulent fluid theory. J Geophys Res 1995; 100: 5723-32.

[6] Hamza A, St-Maurice J-P. A fully self-consistent fluid theory of anomalous transport in Farley-Buneman turbulence. J Geophys Res 1995; 100: 9653-68.

[7] Otani NF, Oppenheim M. A saturation mechanism for the FarleyBuneman instability. Geophys Res Lett 1998; 25(11): 1833-6.
Otani NF, Oppenheim M. Saturation of the Farley-Buneman instability via three-mode coupling. J Geophys Res 2006; 111: A03302.

[9] Dyrud L, Krane B, Oppenheim M, et al. Low-frequency electrostatic waves in the ionospheric E-region: a comparison of rocket observations and numerical simulations. Ann Geophys 2006; 24: 2959-79.

[10] Fejer BG, Providakes J, Farley DT. Theory of plasma waves in the auroral E region. J Geophys Res 1984; 89: 7487-94.

[11] Erukhimov LM, Kagan LM, Savina ON. On thermal mechanism of small-scale irregularity growth in E-layer. Radioph Quant Electronics 1983; 26: 1032.

[12] Gurevich AV, Karashtin AN. Small-scale thermal diffusion instability in the lower ionosphere. Geomag Aeron 1984; 24: 733.

[13] Kissack RS, St.-Maurice J-P, Moorcroft DR. Electron thermal effects on the Farley-Buneman fluid dispersion relation. Phys Plasmas 1995; 2: 1032.

[14] Kissack RS, St.-Maurice J-P, Moorcroft DR. The effect of electronneutral energy exchange on the fluid Farley-Buneman instability threshold. J Geophys Res 1997; 102: 24091-116.

[15] Dimant YS, Sudan RN. Kinetic theory of low-frequency cross-field instability in a weakly ionized plasma. I. Phys Plasmas 1995; 2: 1157-68.

[16] Dimant YS, Sudan RN. Kinetic theory of low-frequency cross-field instability in a weakly ionized plasma, II. Phys Plasmas 1995; 2: 1169-81.

[17] Dimant YS, Sudan RN. Kinetic theory of the Farley-Buheman instability in the E-region of the ionosphere. J Geophys Res 1995; 100: 14605-26.

[18] Dimant YS, Sudan RN. Physical nature of new cross-field instability in the lower ionosphere. J Geophys Res 1997; 102: 2551-63.

[19] Shalimov S, Haldoupis C. An electron thermal diffusion instability and type-3 echoes in the auroral E-region plasma. Ann Geophysicae 1995; 13: 45-55.

[20] Robinson TR. The effects of small-scale field aligned irregularities on E-region conductivities: implications for electron thermal processes. Adv Space Res 1998; 22: 1357-60.

[21] St.-Maurice J-P, Kissack RS. The role played by thermal feedbacks in heated Farley-Buneman waves at high latitudes. Ann Geophys 2000; $18: 532-48$.

[22] Kagan LM, St.-Maurice J-P. Impact of electron thermal effects on Farley-Buneman waves at arbitrary aspect angles. J Geophys Res 2004; 109(A12): A12302.

[23] Kissack RS, Kagan LM, St.-Maurice J-P. Thermal effects on Farley-Buneman waves at nonzero aspect and flow angles. I. Disperssion relation. Phys Plasmas 2008; 15: 022901.

[24] Kissack RS, Kagan LM, St.-Maurice J-P. Thermal effects on Farley-Buneman waves at nonzero aspect and flow angles. II. Behavior near threshold. Phys Plasmas 2008; 15: 022902.

[25] Farley DT, Providakes J. The variation with Te and Ti of the velocity of unstable ionospheric two-stream waves. J Geophys Res 1989; 94(15): 15420-415.

[26] Burgers JM. Flow Equations for Composite Cases; Academic, New York 1969.

[27] St.-Maurice J-P, Choudhary RK, Ecklund WL, Tsunoda RT. Fast type-I waves in the equatorial electrojet: evidence for nonisothermal ion-acoustic speeds in the lower E region. J Geophys Res 2003; 108(5): 1170.

[28] Kudeki E, Farley DT. Aspect sensitivity of equatorial electrojet irregularities and theoretical implications. J Geophys Res 1989; 94: 426-34.

[29] Balsley BB, Farley DT. Radar studies of the equatorial electrojet at three frequencies. J Geophys Res 1971; 76: 8341-51. 
[30] Kagan LM, Kissack RS, Kelley MC, Cuevas R. Unexpected rapid decrease in phase velocity of submeter Farley-Buneman waves with altitude. Geophys Res Lett 2008; 35: L03106.

[31] Leadabrand R, Schlobohm J, Baron M. Simultaneous very high frequency and ultra high frequency observations of the Aurora at Fraserburgh, Scotland. J Geophys Res 1965; 70(17): 4235-84.

[32] Ruohoniemi J, Moorcroft DR. Radar auroral echo heights as seen by a $398-\mathrm{MHz}$ phased array radar operated at Homer, Alaska. Radio Sci 1985; 20(4): 719-34.
[33] Moorcroft DR, Ruohoniemi JM. Nearly simultaneous measurements of radar auroral heights and doppler velocities at 398 MHz. J Geophys Res 1987; 92(A4): 3333-44.

[34] Sahr JD, Fejer BG. Auroral electrojet plasma irregularity theory and experiment: a critical review of present understanding and future directions. J Geophys Res 1996; 101: 26: 893-26909.

[35] Moorcroft DR. The shape of auroral backscatter spectra. Geophys Res Lett 2004; 31: L09802.

(C) Moorcroft et al.; Licensee Bentham Open.

This is an open access article licensed under the terms of the Creative Commons Attribution Non-Commercial License (http: //creativecommons.org/licenses/by$\mathrm{nc} / 3.0 /$ ) which permits unrestricted, non-commercial use, distribution and reproduction in any medium, provided the work is properly cited. 